\title{
Over-expression of CDX2 alleviates breast cancer by up-regulating microRNA let-7b and inhibiting COL11A 1 expression
}

\author{
Hongbin Wang ${ }^{\dagger}$, Yanlv Ren ${ }^{\dagger}$, Cheng Qian, Jiaxin Liu, Ge Li and Zhigao Li
}

\begin{abstract}
Background: microRNA Let-7 serves as a tumor suppressor by targeting various oncogenic pathways in cancer cells. However, the underlying mechanism of its involvement in breast cancer remains largely unknown. With our research, our endeavor is to explore the role of the CDX2/let-7b/COL11A1 axis in breast cancer cell activities.

Methods: Tumor tissues and adjacent normal tissues were collected from 86 patients with breast cancer. Human breast cancer epithelial cell line MCF-7 was treated with over-expressed CDX2, let-7b mimic, shRNA against COL11A1 and their negative controls. The expression of CDX2, let-7b, and COL11A1 in the tissues and cells was determined by RT-qPCR. Interactions among CDX2, let-7b, and COL11A1 were detected by ChIP and dual-luciferase reporter assay, respectively. After different transfections, cell invasion, migration, and proliferation abilities were determined by Transwell and EdU assays. Lastly, tumor xenografts in nude mice were established and hematoxylin and eosin staining was performed to assess the tumor growth and lymph node metastasis.

Results: CDX2 and let-7b were poorly expressed in breast cancer cells and tissues. CDX2 bound to let-7b and promoted the expression of let-7b, which contrarily inhibited the expression of COL11A1. Cancer cell proliferation, invasion, migration, and metastasis were stimulated when CDX2 and let-7b were depleted or COL11A1 was overexpressed. Xenograft tumors growth and metastasis were in accordance with the results of cellular experiments.

Conclusion: In agreement with these observations, we could reach a conclusion that CDX2 could promote let-7b expression, which may exert an inhibitory effect on the proliferation, migration, and metastasis of breast cancer cells via repressing the expression of COL11A1, providing a novel therapeutic strategy for the treatment of metastatic breast cancer.
\end{abstract}

Keywords: Breast cancer, Migration, Invasion, CDX2, let-7b, COL11A1

\section{Background}

Breast cancer is the most common cancer in women, accounting for more than $30 \%$ of new cancer diagnoses in women [1]. Metastasis of breast cancer is a complicated process involving invasion and migration of cancer cells, and blood transversing, and it often associated

*Correspondence: Izg_1964@yeah.net

${ }^{\dagger}$ Hongbin Wang and Yanlv Ren contributed equally to this work

Department of Breast Surgery (No. 2 Sickroom), Harbin Medical University

Cancer Hospital, No. 150, Haping Road, Nangang District, Harbin 150081,

Heilongjiang, People's Republic of China with high mortality rates [2]. Unfortunately, therapies for metastatic breast cancer are limited, and remain to generally incurable [3]. Interestingly, a prior study suggested that breast cancer was affected by gene regulatory networks, in which transcriptional factors and microRNAs (miRNAs) were the most frequently mentioned. Hence, further exploration of the transcriptional factors and miRNAs in the progression of breast cancer might shed some light on prospective diagnoses and management therapies for the highly prevalent malignancy [4].

Transcriptional factors are well-known to play critical roles in numerous human diseases including cancers, 
and regarded as key regulators for new therapies [5]. One such factor, caudal type homeobox 2 (CDX2), a member of the caudal-related homeobox gene family and a mediator of apoptosis in tissues, was found in intestinal epithelial cells [6]. Recently, a study documented reduced CDX2 expression in breast cancer, while its methylation level is elevated [7]. Transcriptional factors have also been demonstrated to play important roles in breast cancer by interacting with miRNAs [8]. MiRNAs, also known as non-coding RNAs have been linked to mediate target genes expression and participate in different cancer cells proliferation, invasion, migration, and metastasis via several signaling pathways [2]. The let-7b was frequently reported with a decreased expression in cancers. For instance, the let- 7 family members (let-7a, let-7b, and let-7g) have been demonstrated to be poorly-expressed in the patients diagnosed with breast cancer with lymph node metastasis [9]. Previous work further illustrated the targeting relationship between miRNA and collagen in breast cancer [10]. Interestingly, through bioinformatics and a dual luciferase reporter gene assay, COL3A1 was suggested as a direct target of let-7b in neuronal injury [11]. Collagen was reported to be active in numerous biological processes in cancers [12]. Collagen type XI alpha 1 chain (COL11A1), a subtype of fibrillary type collagen, plays a role in extracellular matrix and tissues and was over-expressed in human tumors [13]. COL11A1 has also been regarded as a promising prognostic biomarker for breast cancer [13]. On the basis of existing literature data, we carried out studies in an effort to investigate the possible CDX2 may act as a significant cytokine in breast cancer. This study distinguishes from other studies by emphasizing the role of transcriptional factors and miRNAs in the invasion, migration, and metastasis of breast cancer epithelial cells.

\section{Methods and materials}

\section{Ethical statement}

All patients participated in this study signed informed consent and the study protocols were approved by the Ethics Committees of Harbin Medical University Cancer Hospital. All animal experiments in this study have been approved by the Ethics Committee of Harbin Medical University Cancer Hospital. The animals received humane care according to the Guide for the Care and Use of Laboratory Animals published by the US National Institutes of Health.

\section{Breast cancer tissue samples}

Breast cancer tissue samples were collected from 86 patients (49 cases aged $<50$ years old; 37 cases aged $\geq 50$ years old) undergoing surgical resection of breast cancer at the Harbin Medical University Cancer Hospital between April 2014 and August 2017. In addition, adjacent normal tissue samples were also resected from all patients as controls. The samples used were not subjected to preoperative radiotherapy and/ or chemotherapy, and were diagnosed as non-specific invasive breast cancer by more than two associate chief physicians at the Department of Pathology. After diagnoses, the samples were classified according to World Health Organization tumor classification standard. There were 51 samples classified as grade I+ II (benign and atypical), and 35 samples classified as grade III (anaplastic). Next, the tumors were evaluated based on the sixth edition of the American Joint Committee on Cancer tumor, node, metastases staging system (stage $\mathrm{I} / \mathrm{II}, \mathrm{n}=57$; stage III, $\mathrm{n}=29$ ). No lymphatic metastasis was found in the samples, which were fixed with $10 \%$ formalin, dehydrated, paraffin-embedded and sectioned into $4 \mu \mathrm{m}$ for further experimentation.

\section{Immunohistochemistry}

Paraffin-sectioned tissues sections were dewaxed and dehydrated. The target antigens were retrieved by microwaving the sections in Tris-ethylenediaminetetraacetic acid (EDTA) buffer containing $10 \mathrm{mM}$ Tris and $1 \mathrm{mM}$ EDTA $(\mathrm{pH}=9.0)$ The samples were heated in a microwave at $800 \mathrm{~W}$ for $3 \mathrm{~min}$ and then for $7 \mathrm{~min}$ at $400 \mathrm{~W}$ to continue boiling. The sections were cooled down for $30 \mathrm{~min}$ at room temperature. After that, the 10-min heating procedure was repeated. After being blocked with normal goat serum (C-0005, Shanghai Haoraobio Science \& Technology Co., Ltd., Shanghai, China), the tissues were incubated with the primary rabbit anti-human antibody to CDX2 (dilution ratio of 1:100, ab88129) and secondary goat anti-rabbit immunoglobulin G (IgG; dilution ratio of 1:1000, ab6785) at $37^{\circ} \mathrm{C}$ for $20 \mathrm{~min}$, followed by incubation with horseradish peroxidase (HRP)-conjugated Streptavidin (034310000 U, Imunbio Co., Ltd, Beijing, China) at $37{ }^{\circ} \mathrm{C}$ for $20 \mathrm{~min}$. Next, the tissues were developed using diaminobenzidine (ST033, Whiga Biosmart Co., Ltd., Guangzhou, Guangdong, China), counterstained with hematoxylin (PT001, Shanghai Bogoo Biotechnological Co., Ltd., Shanghai, China) for $1 \mathrm{~min}$, and returned to blue using $1 \%$ ammonium hydroxide, mounted and observed by microscopy. Five high power field of vision were randomly selected from each slice with 100 cells in per field of vision. Sections with less than $10 \%$ positive-cells were regarded as negatively expressed, more than $10 \%$ and less than $50 \%$ were considered as positively expressed, and more than $50 \%$ were considered as strongly positively expressed. 


\section{Cell culture and treatment}

Normal human breast epithelial cell line HBL-100, and human breast cancer epithelial cell lines MCF-7 and MDA-MB-231 were procured from the Cell Bank of Chinese Academy of Sciences (Shanghai, China), and the cell line exhibiting the lowest expression of CDX2 was selected using RT-qPCR.

Subsequently, plasmids of over-expressed CDX2, let7b mimic, let-7b inhibitor, COL11A1 over-expression, shRNA targeting COL11A1 and their relevant negative control23 (NC), and lentiviral expression vectors of pGCFU-GFP and pLVX-shRNA were constructed by Sangon Biotech (Shanghai, China). Cells at passage three were transfected with the above-mentioned plasmids using Lipofectamine 2000 (Invitrogen, Carlsbad, CA, USA). Next, the stably transfected cells were screened and cultured in the medium with G418 (1000-2000 $\mu \mathrm{g} / \mathrm{mL})$ for 4 weeks.

\section{RNA isolation, reverse transcription, and quantitative real-time polymerase chain reaction}

Total RNA content was extracted from breast cancer and adjacent normal tissues using the TRIZOL reagent (15596-018, Beijing Solarbio Science \& Technology Co., Ltd., Beijing, China) and then reverse transcribed into complementary DNA (cDNA) in strict accordance with the manufacturer's protocols of the kit (K1622, Beijing Reanta Biotechnological Co., Ltd, Beijing, China). Primers were synthesized by Takara Bio (Dalian, Liaoning, China) (Table 1). Glyceraldehyde-3-phosphate dehydrogenase (GAPDH) was regarded as the internal reference primer and the relative transcriptional levels of the target genes were calculated using the $2^{-\Delta \Delta \mathrm{Ct}}$ method.

\section{Western blot}

After isolation from tissues and cell, protein was then quantified with bicinchoninic acid assay protein assay kit (20201ES76, Yeasen Biotechnology Co., Ltd., Shanghai, China). After quantification, the proteins were separated using polyacrylamide gel electrophoresis and transferred onto a polyvinylidene fluoride membrane. Subsequently, the membrane was hybridized with primary rabbit antihuman antibodies to CDX2 (dilution ratio of 1:2000, ab88129), COL11A1 (dilution ratio of 1:1000, ab64883), E-cadherin (dilution ratio of 1:500, ab15148), Vimentin (dilution ratio of 1:2500, ab92547) and GAPDH (dilution ratio of 1:2500, ab9485) and with the goat anti-rabbit IgG labeled by HRP (dilution ratio of 1:20,000, ab205718). All aforementioned antibodies were purchased from Abcam Inc. (Cambridge, UK). After the membrane was developed, protein quantitative analysis was performed using the ImageJ 1.48u software (National Institutes of Health, Bethesda, MA, USA).

\section{Chromatin immunoprecipitation (ChIP)}

MCF-7 cells were fixed with formalin for $10 \mathrm{~min}$ to allow crosslinking of DNA and proteins. The ultrasonic breaker was set to $10 \mathrm{~s}$ per ultrasonic cycle with 10 -s intervals with 15 cycles to break the chromatin. Then, the supernatant was harvested and divided into two tubes, one being supplemented with IgG (ab6785, Abcam) and the other added with target protein-specific antibodies to CDX2 (sc-134362, $2 \mu \mathrm{G} / 1 \mathrm{~mL}$ cell lysate, Santa Cruz Biotechnology Inc, Santa Cruz, CA, USA) respectively. Next, the DNA-protein complex was precipitated using Protein Agarose/Sepharose, followed by de-crosslinking. DNA fragments were extracted and purified using a phenol/ chloroform mixture. Finally, the binding of let-7b promoter region was detected using specific primers of the let-7b promoter.

\section{Dual-luciferase reporter gene assay}

According to the sequence of let-7b mRNA promoter region binding to CDX2, let-7b promoter wild type (WT) sequence and mutation (Mut) sequence were designed. Simultaneously, MCF-7 cells were plated on a 24-well plate. The luciferase reporter plasmids COL11A1-WTLuc and COL11A1-Mut-Luc were co-transfected with let-7b mimic or $\mathrm{NC}$ of let-7b mimic into the MCF-7 cells upon reaching $80 \%$ confluence. At $48 \mathrm{~h}$ after transfection of the reporter plasmid, the cells were harvested and reporter assays were performed using a dual luciferase reporter assay system (D0010, Beijing Solarbio Science \& Technology Co., Ltd, Beijing, China). The fluorescence intensity was determined using a GLomax20/20

Table 1 Primer sequences for RT-qPCR

\begin{tabular}{|c|c|c|}
\hline Genes & Forward primer sequences $\left(5^{\prime}-3^{\prime}\right)$ & Reverse primer sequences $\left(5^{\prime}-3^{\prime}\right)$ \\
\hline CDX2 & CCACGCTGGGGCTCTCT & GTCTAGCAGAGTCCACGCTC \\
\hline let-7b & TGAGGTAGTAGGTTGTGTGGTT & GCGAGCACAGAATTAATACGAC \\
\hline GAPDH & ACCTGACCTGCCGTCTAGAA & TCCACCACCCTGTTGCTGTA \\
\hline COL11A1 & TGGTGATCAGAATCAGAAGTTCG & AGGAGAGTTGAGAATTGGGAATC \\
\hline
\end{tabular}

CDX2, caudal type homeobox 2; let-7b, microRNA let-7b; COL11A1, collagen type XI alpha 1 chain; GAPDH, glyceraldehyde-3-phosphate dehydrogenase 
Luminometer (Promega Corporation, Madison, WI, USA).

\section{5-ethynyl-2'-deoxyuridine (EdU) staining}

MCF-7 cells seeded in 96-well plates at a density of $5 \times 10^{4}$ cells/well were transfected with miRNA as described. Proliferation assays were performed $48 \mathrm{~h}$ after transfection in accordance with the manufacturer's protocol. In brief, after incubation with medium containing EdU for $2 \mathrm{~h}$, the cells were detached, fixed with 4\% paraformaldehyde for $30 \mathrm{~min}$ and incubated with reagents of $\mathrm{B}, \mathrm{C}, \mathrm{D}$, and $\mathrm{E}$ based on the EdU kit protocols (Guangzhou RiboBio Co., Ltd., Guangzhou, Guangdong, China). The cells were finally incubated with Hoechst33342 staining solution and then photographed using fluorescence microscopy. Cells presenting with red-stained nucleus were regarded as positive cells. EdU labeling rate calculations were performed as previously described [7].

\section{Transwell assay}

MCF-7 cells were resuspended and seeded evenly in a 24-well plate (density of $3 \times 10^{4}$ cells/well), with $200 \mu \mathrm{L}$ culture medium added to the apical chamber, and complete media in the basolateral chamber wells serving as a chemo-attractant. Subsequently, the plates were incubated with $\mathrm{CO}_{2}$. At $24 \mathrm{~h}$, cells on the upper surface of the membrane were removed using a cotton swab, and the basolateral chamber was fixed with $800 \mu \mathrm{L}$ mixture of methanol-glacial acetic acid at a ratio of 3:1 for $20 \mathrm{~min}$. Transwell chambers were then stained with $500 \mu \mathrm{L}$ crystal violet for $5 \mathrm{~min}$. The stained cells were photographed and counted under an optical microscope. Subsequently, the average number of cells was calculated and recorded.

Matrigel stored at $-20{ }^{\circ} \mathrm{C}$ was melted and diluted with pre-cooled serum-free medium at a ratio of 1:9. Next, Transwell apical chamber was seeded in a 24-well plate and coated with a total of $50 \mathrm{~L}$ diluted Matrigel. Transwell invasion assay was carried out following a similar procedure as the Transwell migration assay. The number of cells that invaded the membrane was measured and recorded.

\section{Tumor xenografts in nude mice}

BALB/c nude mice (aged 5 to 7 weeks; weighing 19-21 g) were purchased from Shanghai Lingchang Biological Technology Co., Ltd. (Shanghai, China) and raised under specific-pathogen-free conditions. The nude mice were then assigned into different groups with 8 mice per group and inoculated with transfected MCF-7 cells.

The cells were dispersed into a cell suspension $\left(5 \times 10^{7}\right.$ cells $/ \mathrm{mL}$ ). Nude mice were subcutaneously injected with $0.2 \mathrm{~mL}$ cell suspension in the left armpits. Then, tumor growth was recorded on the 7th, 14th, 21st and 28th day after injection using Vernier calipers. Tumor volume was calculated 3 times with the formula $\pi(2 \mathrm{ab}) / 6$, in which a referred to the short diameter, while $b$ was the long diameter. The mice were euthanized on the 28 th day and tumors were extracted. Tumor tissues were then fixed with $10 \%$ formalin, dehydrated, paraffin-embedded and serially cut into $4-\mu \mathrm{m}$ thick sections for RNA and protein content extraction. Meanwhile, hematoxylin and eosin (H\&E) staining of the lungs and livers was performed in order to observe lymph node metastasis.

\section{Statistical analyses}

All measurement data were shown as mean \pm standard deviation and analyzed using the SPSS 21.0 software (IBM Corp., Armonk, NY, USA). Comparisons of statistics with normal distribution and uniformed variance between two groups were analyzed using the paired $t$ test or unpaired $t$-test. One-way analysis of variance (ANOVA) was performed for comparisons among groups followed by Tukey's post hoc test. Repeated measures ANOVA was carried out for comparisons among groups at different time points followed by Bonferroni post hoc test. A value of $p<0.05$ was considered to be statistically significant.

\section{Results}

CDX2 is poorly expressed in breast cancer, while over-expressed CDX2 inhibits migration and invasion of breast cancer epithelial cells

As compared to adjacent normal tissues, CDX2 expression was decreased in breast cancer tissues, and the CDX2 protein was located at the nucleus and stained in brown $(p<0.05$; Fig. $1 \mathrm{a}-\mathrm{c})$. Western blot analysis demonstrated that the CDX2 protein was poorly expressed in breast cancer tissues (Fig. 1d). Analysis of survival rate revealed that down-regulation of CDX2 was evident in patients with low survival rates (Fig. 1e).

Furthermore, the expression of CDX2 was diminished in human breast cancer epithelial cell lines MCF-7 and MDA-MB-231 compared to that of normal human breast epithelial cell line HBL-100, where the MCF-7 cell line exhibited the lowest expression of CDX2 $(p<0.05$; Fig. 1f). Thereby, MCF-7 cells were chosen for further experimentation.

Subsequently, Fig. 1g demonstrated that oe-CDX2 was successfully delivered, and further experiments were continued. In addition, results from EdU assay revealed that EdU positive-cells treated with over-expressed CDX2 showed a marked decrease compared to the positive-cells in NC of over-expressed CDX2 ( $p<0.05$; Fig. 1h). Moreover, over-expression of CDX2 inhibited the migration and invasion of MCF-7 cells $(p<0.05$; Fig. 1i, j). Western blot results further demonstrated that CDX2 and E-cadherin 


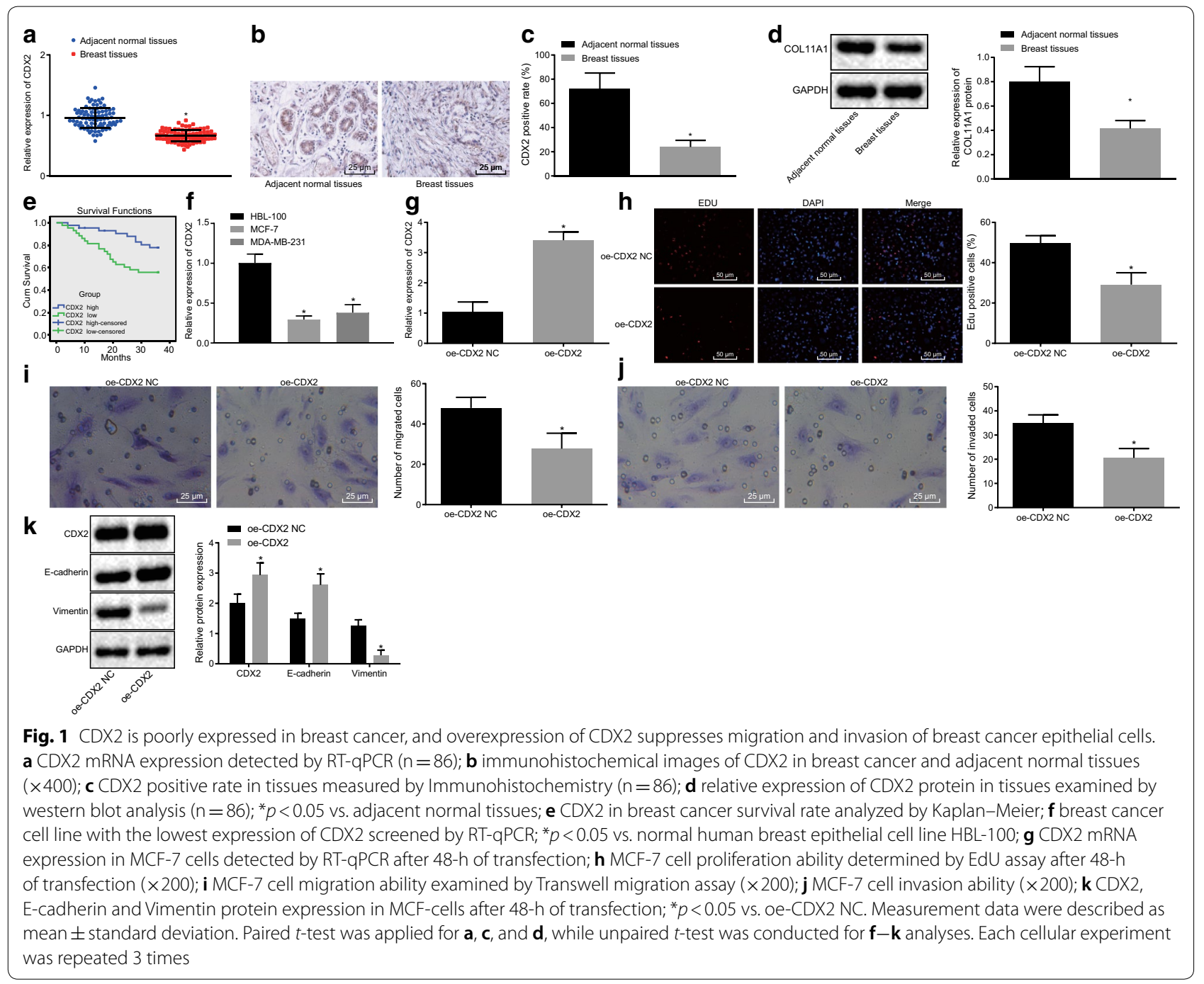

protein expression was enhanced in cells treated with over-expressed CDX2, while the protein expression of Vimentin showed a significant decline $(p<0.05$; Fig. $1 \mathrm{k})$ compared to the expression in $\mathrm{NC}$ of over-expressed CDX2. These results collaboratively illustrated that overexpressed CDX2 inhibited the proliferation, migration, and invasion abilities of breast cancer epithelial cells.

\section{CDX2 inhibits migration and invasion of breast cancer epithelial cells by up-regulating let-7 $b$}

TransmiR database and the relevant microarray of breast cancer, GSE45666 (Fig. 2a, b) indicated that CDX2 may mediate the let-7b. Subsequent RT-qPCR displayed that the expression of let-7b was decreased in breast cancer tissues in contrast to adjacent normal tissues $(p<0.05$; Fig. $2 \mathrm{c})$. The CDX2 promoter region in let-7b was identified using ChIP assay, and results confirmed that CDX2 was enriched in let-7b (Fig. 2d). Meanwhile, dual-luciferase reporter gene assay results demonstrated that the luciferase activity of cells transfected by overexpressed CDX2 and let-7b WT was visibly enhanced when compared with NC $(p<0.05)$. However, no significant differences were detected in luciferase intensity in the Mut plasmid ( $p>0.05$; Fig. 2e).

Subsequently, RT-qPCR was carried out to evaluate the efficiency of transfection, which substantiated that the delivery was successful $(p<0.05)$. After co-transfection with CDX2 over-expressed plasmid, let-7b mRNA was found to be highly expressed in cells treated with inhibited let-7b and over-expressed CDX2 when compared with the relevant NC ( $p<0.05$; Fig. $2 f)$.

Furthermore, results obtained from EdU, Transwell and western blot analysis (Fig. $2 g-j$ ) revealed that EdU positive-cells were decreased, cell migration and invasion abilities were suppressed, E-cadherin proteins were increased while Vimentin protein expression was 


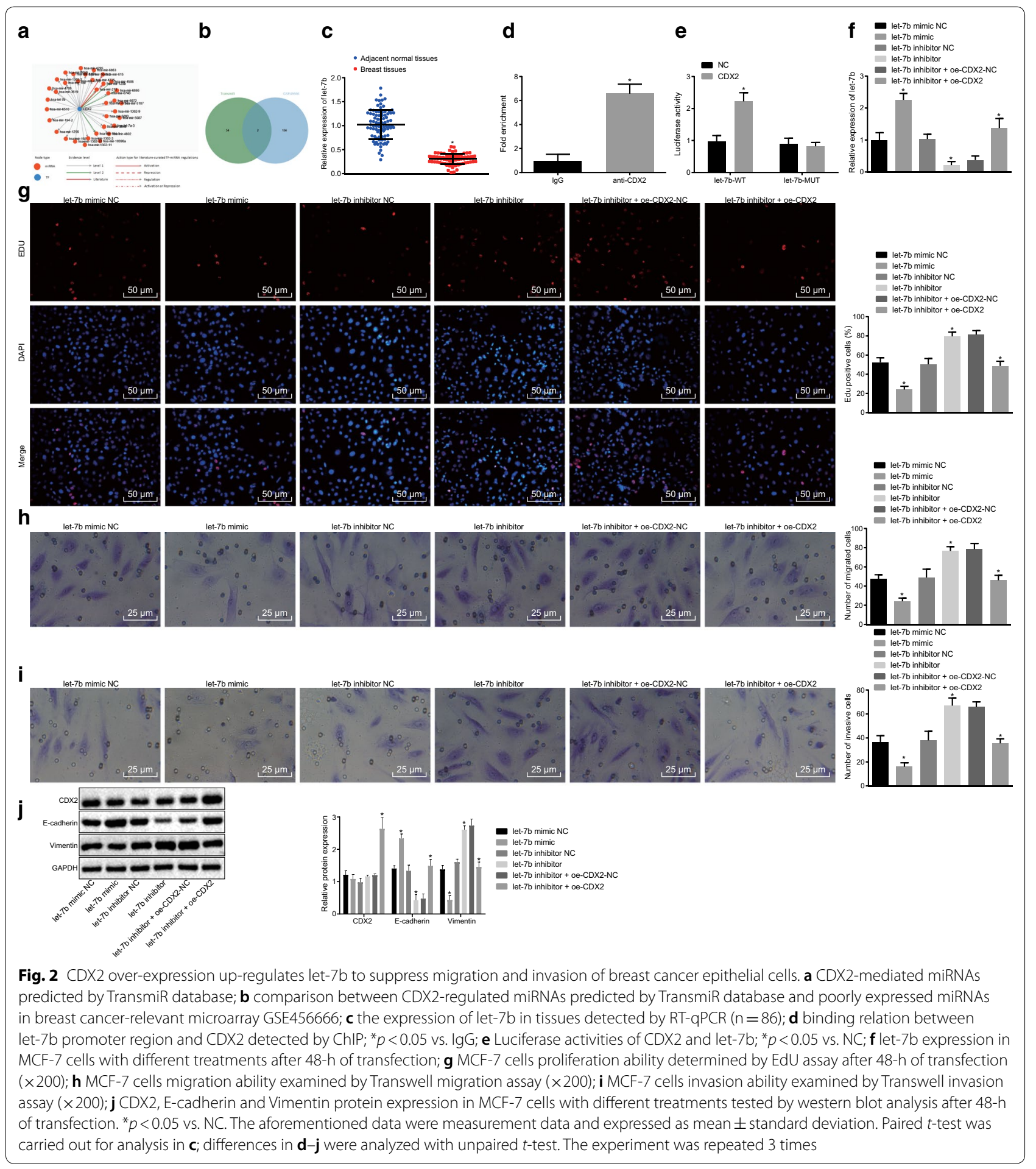

significantly reduced in let-7b mimic-treated MCF-7 cells when compared with its NC $(p<0.05)$. However, let-7b mimic had no impact on the protein expression of CDX2 $(p>0.05)$. The results were reciprocal in the comparisons between the let-7b inhibitor and its $\mathrm{NC}$ with unchanged protein expression of CDX2 $(p>0.05)$. Meanwhile, in cells treated with both let-7b inhibitor and CDX2 overexpression, EdU positive-cells were reduced, cancer cells migration and invasion were restrained, E-cadherin and CDX2 protein expression was increased and Vimentin 
protein expression was decreased relative to $\mathrm{NC}$ of let-7b inhibitor and over-expressed CDX2 $(p<0.05)$. These data showed that over-expression of CDX2 up-regulated the expression of let- $7 \mathrm{~b}$, consequently inhibiting the migration and invasion of breast cancer epithelial cells.

\section{Let- $7 \mathrm{~b}$ reduces breast cancer cell migration and invasion by directly down-regulating $\mathrm{COL} 11 \mathrm{~A} 1$}

In order to realize the target genes of let-7b, highlyexpressed genes in breast cancer were predicted using the TargetScan database (Fig. 3a) and breast cancerrelated microarrays including GSE109169, GSE3744, and GSE26910, which suggested that the COL11A1 gene may be a target of let-7b. Meanwhile, the binding sites between let-7b and COL11A1 were predicted using the TargetScanHuman website (http://www.targetscan.org/; Fig. 3b). RT-qPCR demonstrated that COL11A1 was highly expressed in cancer tissues compared to adjacent normal tissues $(p<0.05$; Fig. $3 c)$. The results of dualluciferase reporter gene assay revealed that the luciferase activity in co-transfection of let-7b mimic and COL11A1 WT was remarkably decreased when compared with its $\mathrm{NC}(p<0.05)$, while the luciferase activity in Mut plasmid displayed no obvious differences ( $p>0.05$; Fig. $3 \mathrm{~d})$.

Next, RT-qPCR was conducted to assess the overexpression or silencing efficiency of delivery (Fig. 3e). Results demonstrated that COL11A1 expression was increased when the cells were treated with overexpressed COL11A1 compared with its NC $(p<0.05)$, while COL11A1 expression was declined in cells treated with sh-COL11A1 in contrast to that in NC of shCOL11A1 $(p<0.05)$. Meanwhile, the results obtained from EdU, Transwell and western blot analysis (Fig. 3f-i) revealed that EdU positive-cells were increased, migration and invasion were promoted, COL11A1 and Vimentin protein expression was enhanced and the protein expression of E-cadherin was decreased in MCF-7 cells treated with over-expressed COL11A1 compared with that in NC of over-expressed COL11A1. MCF-7 cells treated with sh-COL11A1 showed reduced EdU positivecells, migration and invasion as well as COL11A1 and Vimentin protein expression, while enhanced E-cadherin protein expression relative to sh-COL11A1 NC $(p<0.05)$.

Furthermore, cells with over-expressed COL11A1 were co-transfected with let-7b mimic plasmid to investigate the regulatory mechanism between let-7b and COL11A1. Results from RT-qPCR illustrated that the expression of COL11A1 was diminished after co-transfection with over-expressed COL11A1 and let-7b mimic compared to its NC (Fig. 3j). Results from EdU, Transwell and western blot analysis (Fig. $3 \mathrm{k}-\mathrm{n}$ ) revealed that when MCF-7 cells were treated with over-expressed COL11A1 and let-7b mimic, EdU positive-cells, cells migration and invasion abilities as well as the protein expression of COL11A1 and Vimentin were down-regulated, while the protein expression of E-cadherin was increased when compared to the $\mathrm{NC}$ of over-expressed COL11A1 and let-7b mimic $(p<0.05)$. These data strongly indicated that let-7b may downregulate breast cancer cell migration and invasion via direct repression of COL11A1.

\section{CDX2/let-7b/COL11A1 modulates growth and metastasis of breast cancer in vivo}

On the one hand, MCF-7 cells were subcutaneously injected into the left armpits of nude mice, which were randomized into six groups. We found that both overexpression of $\mathrm{CDX} 2$ or let-7b, or down-regulation of COL11A1 efficiently reduced the volume and weight of the tumor, as shown in Fig. $4 \mathrm{a}-\mathrm{c}(p<0.05)$.

Additionally, lung and liver tissues were extracted from nude mice and made into paraffin samples for lymph node metastasis analysis. H\&E staining was employed to examine lymph node metastasis and results demonstrated that the irregular cancer cells of lung and liver tissues and other poorly differentiated characteristics were observed (Fig. 4e). Compared to the NC of CDX2 over-expression vector, positive lymph node metastasis was observed in 2 out of 8 nude mice inoculated with

\footnotetext{
(See figure on next page.)

Fig. 3 Let-7b reverses the effect of COL11A1 over-expression on breast cancer cell migration and invasion. a let-7b target genes predicted by TargetScan database and highly expressed genes in microarrays GSE109169, GSE3744 and GSE26910; b binding sites of let-7b and COL11A1; $\mathbf{c}$ COL11A1 mRNA expression in clinical tissues detected by RT-qPCR $(n=86) ; \mathbf{d}$ Luciferase activity of let-7b and COL11A1; ${ }^{*} p<0.05$ vs. NC; e COL11A1 mRNA expression in MCF-7 cells treated with depleted COL11A1 determined by RT-qPCR after 48-h of transfection; $\mathbf{f}$ proliferation of MCF-7 cells treated with silenced COL11A1 expression detected by EdU assay after 48-h of transfection ( $\times 200) ; \mathbf{g ~ M C F - 7 ~ c e l l ~ m i g r a t i o n ~ a b i l i t y ~ a f t e r ~ C O L 1 1 A 1 ~}$ knockdown determined by Transwell migration assay ( $\times 200)$; $\mathbf{h}$ MCF-7 cell invasion ability after COL11A1 knockdown examined by Transwell invasion assay ( $\times 200$ ); $\mathbf{i}$ CDX2, E-cadherin and Vimentin protein expression in MCF-7 cells treated with silenced COL11A1 detected by western blot analysis after 48-h of transfection; j COL11A1 mRNA expression in MCF-7 cells with different treatments determined by RT-qPCR after 48-h of transfection; k MCF-7 cells proliferation tested by EdU assay after 48-h of transfection ( $\times 200)$; I MCF-7 cell migration ability $(\times 200) ; \mathbf{m ~ M C F - 7 ~}$ cells invasion ability ( $\times 200) ; \mathbf{n}$ CDX2, E-cadherin and Vimentin protein expression in MCF-7 cells detected by western blot analysis after 48-h of transfection. ${ }^{*} p<0.05$ vs. NC. The above statistics were measurement data, which were expressed as mean \pm standard deviation. Except for $\mathbf{c}$ which was analyzed by paired $t$-test, analyses in $\mathbf{d}$-n were carried out using unpaired $t$-test. Each cellular experiment was performed at least 3 times
} 


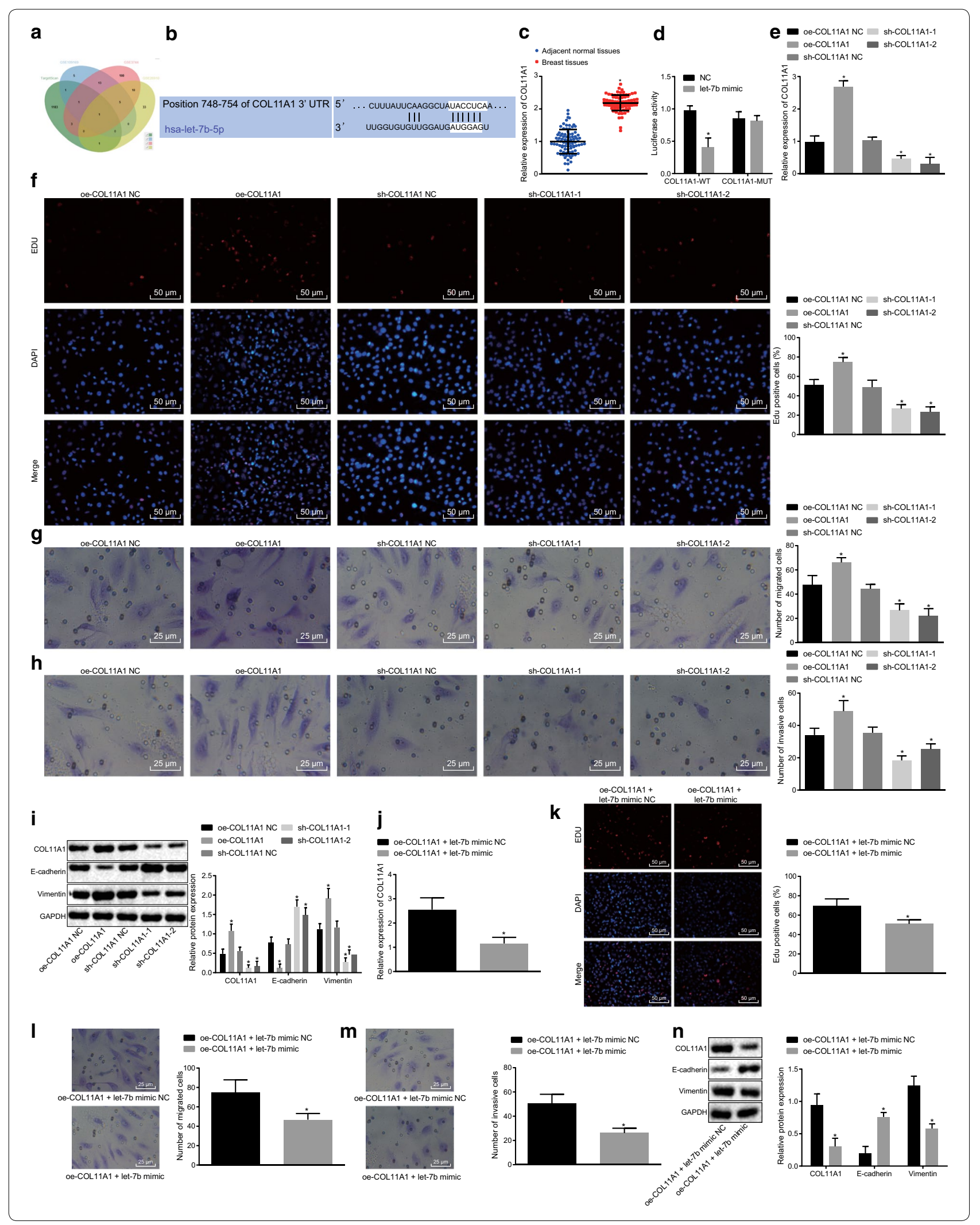




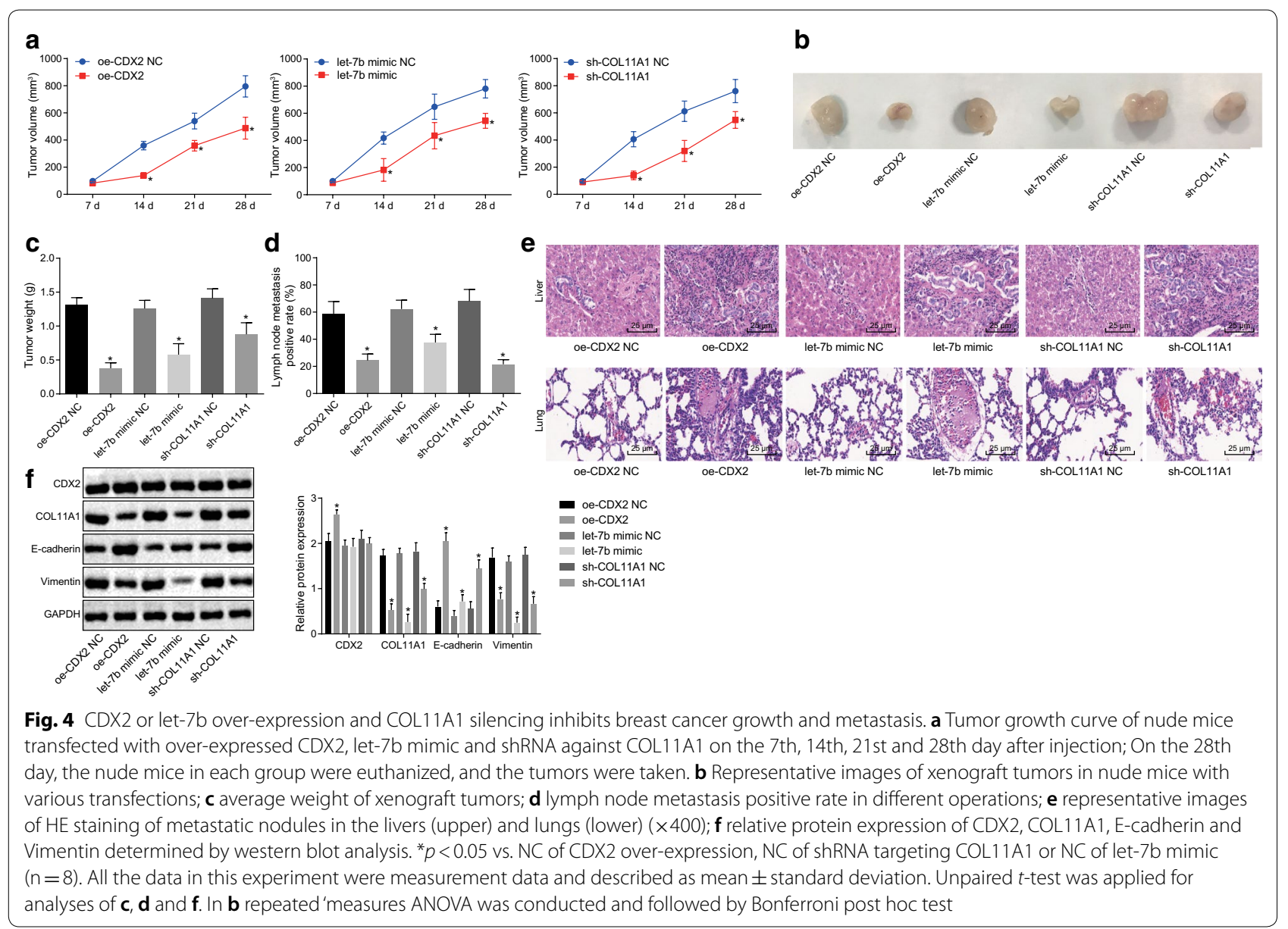

over-expressed CDX2 (positive metastasis rate $=24.35 \%$ ). $H \& E$ staining further showed that the tumor cells were decreased in response to treatment with CDX2 overexpression $(p<0.05)$. In contrast to $\mathrm{NC}$ of let-7b mimic, positive lymph node metastasis was observed in 3 out of 8 nude mice (with let-7b treatment) where the rate of positive metastasis was $37.5 \%$, and tumor cell number was also evidently reduced $(p<0.05)$. Tumor cells were likewise decreased in nude mice inoculated with shRNA against COL11A1, where the positive metastasis rate was $25 \%$ as 2 out of 8 nude mice presented positive lymph node metastasis compared to NC of shRNA against COL11A1 (Fig. 4d).

Western blot analysis (Fig. 4f) demonstrated that after treatment with over-expressed CDX2, the expression of E-cadherin was increased, while the expression of COL11A1 and Vimentin was decreased compared with its relevant NC $(p<0.05)$. Similarly, E-cadherin expression was also enhanced while the expression of COL11A1 and Vimentin was reduced upon treatment with let-7b mimic in contrast to that of its $\mathrm{NC}(p<0.05)$. When treated with shRNA targeting COL11A1, CDX2 showed no significant difference $(p>0.05)$, the expression of E-cadherin was elevated, while the expression of COL11A1 and Vimentin was reduced compared with NC of shRNA against COL11A1 $(p<0.05)$. These data indicated that let-7b or CDX2 up-regulation or COL11A1 down-regulation can inhibit breast cancer growth and metastasis.

\section{Discussion}

Tumor cells metastases led by tumor cell invasion and migration are regarded as the most fatal stage in cancers. For example, breast cancer metastases can be widely disseminated to several regions in the human body including the brain, bone, liver, and lung [14]. Furthermore, metastasis often occurred before diagnosis in breast cancer [15]. Therefore, it is trivial to seek effective prognostic markers to predict metastasis in breast cancer. Burgeoning genetic researches have been widely reported on tumor cells invasion, migration and metastasis. A previous study highlighted the importance of genetic factors elucidation in the pathogenesis of breast cancer [16]. However, limited studies have explored the 
gene regulatory networks implicated in breast cancer. In the current study, we proposed that breast cancer metastasis was correlated with CDX2, and CDX2 could inhibit breast cancer progression by up-regulating let- $7 \mathrm{~b}$.

Initially, we found that CDX2, which was frequently studied in intestinal cancer, was associated with the survival of patients with breast cancer. Our findings further indicated that CDX2 was poorly expressed in breast cancer, while the over-expressed CDX2 diminished the migration and invasion abilities of breast cancer epithelial cells. As a homeotic transcriptional factor, CDX2 was poorly expressed in colon cancer and associated with invasion and metastasis of cells [17]. Therefore, it would be reasonable to suggest that poor expression of CDX2 is correlated with migration and invasion of breast cancer epithelial cells, and could be countered by over-expression of CDX2.

A previous study has demonstrated that miRNAs were regulated by CDX2 [18]. Similarly, we uncovered that CDX2 inhibited the migration and invasion of breast cancer epithelial cells by up-regulating let-7b. Let-7b has also been previously highlighted as a tumor suppressor due to its involvement in various mechanisms, including cell proliferation and invasion [19]. Similarly, another study displayed that let-7b was poorly expressed in patients diagnosed with breast cancer at clinical stages I-IV [20]. In addition, our data demonstrated that let-7b mimic transfection resulted in the inhibition of cells invasion and migration, while poor prognoses were occurred in breast cancer patients with reduced let-7b expression, suggesting that let-7b may be a potential biomarker for breast cancer cell behavior. With regard to regulation of miRNAs in cancers, accumulating evidence put emphasis on the specific miRNA-regulated mechanisms and indicated that let-7b could serve as an effective index in distinguishing breast cancer clinical behaviors [21]. Furthermore, He et al. found that CDX2 bound to the promoter region and inhibited miR-145-5p transcription, thus relieving the suppressive effect of miR-145-5p on the translation of small ubiquitin-like modifier proteinspecific protease 1 and affecting the invasion and migration of prostate cancer cells [22]. In the present study, we first predicted the targeting relationship between CDX2 and let-7b using the TargetScan website with breast cancer microarray data, and examined the binding of CDX2 to the let-7b promoter region with ChIP assay. Based on these experiments, it was confirmed that CDX2 targets let- $7 \mathrm{~b}$. These findings highlight the gene regulatory network, where genes and miRNAs were intertwined in breast cancer epithelial cells activities.

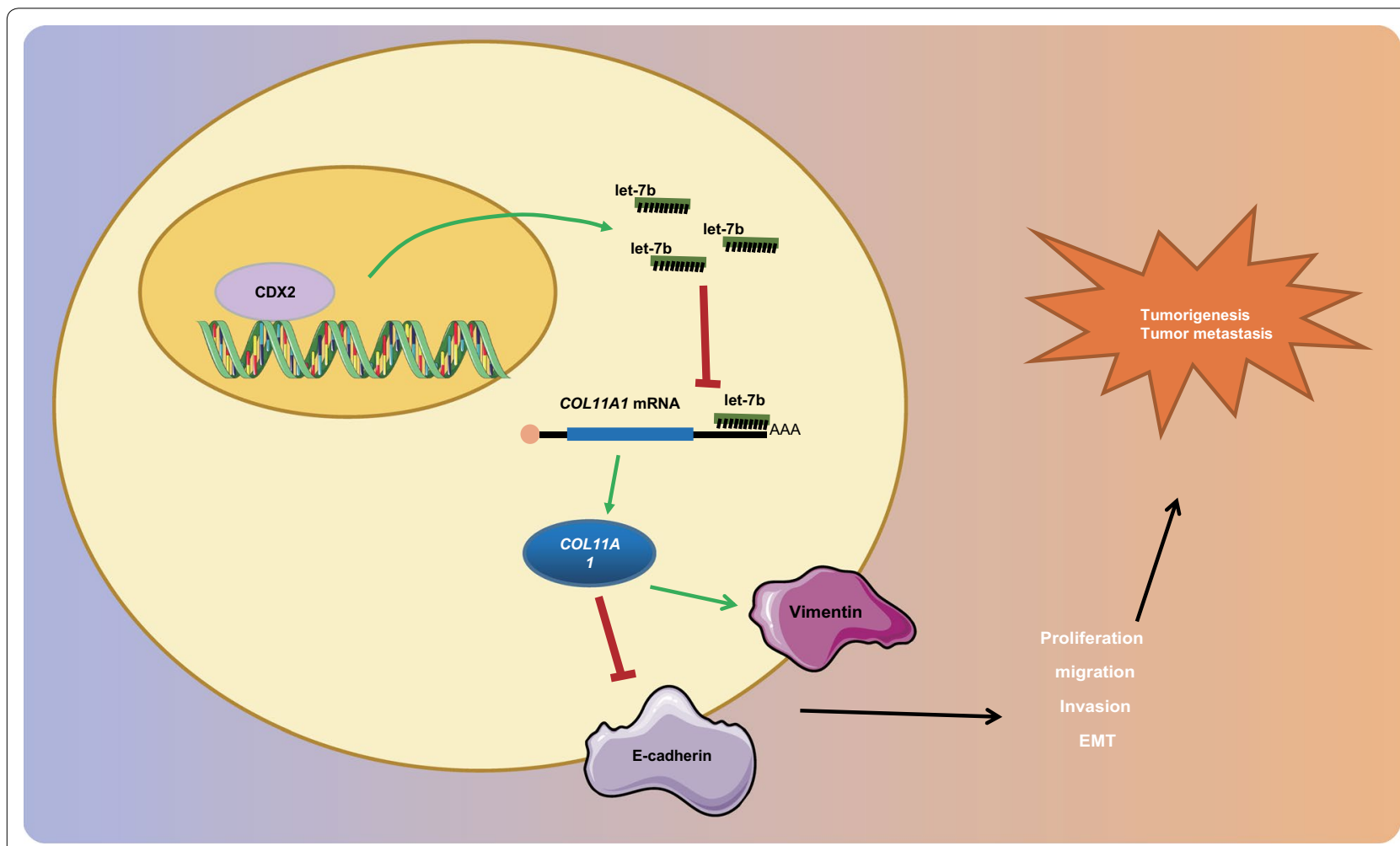

Fig. 5 CDX2 promotes the expression of let-7b and inhibits COL11A1, thus suppressing the proliferation, invasion, and migration abilities of breast cancer cells 
Furthermore, we explored the target genes of let-7b in the current study. The COL11A1 gene was screened using the TargetScan database, and subsequently, the binding sites between let-7b and COL11A1 were predicted by the TargetScanHuman website. The targeting relationship was substantiated by a dual luciferase reporter gene assay. Meanwhile, a prior research reported that COL11A1 was highly expressed in cancers and potentiated the invasion by coordinating with invasion-sensing biomarkers. However, there were no reports on the direct association between COL11A1 and breast cancer in the study [23]. Another study unraveled that COL11A1 expression was found in fibroblasts associated with breast cancer and played a vital role in the metastasis of tumor cells [24]. In the present study, over-expressed CDX2 and let-7b inhibited lymph node metastasis and tumor growth by silencing COL11A1.

\section{Conclusion}

Overall, the current study verified the CDX2/let-7b/ COL11A1 modulation in breast cancer epithelial cells, and investigated the tumor growth and metastasis through in vivo models (Fig. 5). Our findings highlight the role of gene regulatory networks in tumor cell metastasis, which unlocks novel avenues for breast cancer therapy. However, more in-depth investigations and optimization are needed in future studies to ensure the successful therapeutic biomarker discovery for the treatment and prognosis of breast cancer.

\section{Abbreviations \\ CDX2: caudal type homeobox 2; COL11A1: collagen type XI alpha 1 chain; IHC: immunohistochemistry; NC: negative control; cDNA: complementary DNA; GAPDH: glyceraldehyde-3-phosphate dehydrogenase; ChIP: chromatin immu- noprecipitation; WT: wild type; Mut: sequence and mutation; EdU: 5-ethynyl- 2'-deoxyuridine; ANOVA: analysis of variance.}

\section{Acknowledgements}

We would like show sincere appreciation to the colleagues for their assistance on this study.

\section{Authors' contributions}

$H W, Y R, C Q, G L$ and $J L$ wrote the paper and conceived and designed the experiments; HW, YR, ZL and JL analyzed the data; CQ, ZL and GL collected and provided the sample for this study. All authors read and approved the final manuscript.

\section{Funding}

This study was supported by Harbin Applied Technology Research and Development Project Plan (Harbin Science and Technology Bureau) (No. 2016RAQXJ174).

\section{Availability of data and materials}

The datasets generated/analyzed during the current study are available.

\section{Ethics approval and consent to participate}

All patients participated in this study signed informed consent and the study protocols were approved by the Ethics Committees of Harbin Medical
University Cancer Hospital. All animal experiments in this study have been approved by the Ethics Committee of Harbin Medical University Cancer Hospital. The animals received humane care according to the Guide for the Care and Use of Laboratory Animals published by the US National Institutes of Health.

\section{Consent for publication}

Not applicable.

\section{Competing interests}

The authors declare that they have no competing interests.

Received: 6 August 2019 Accepted: 10 December 2019

Published online: 10 January 2020

\section{References}

1. Siegel RL, Miller KD, Jemal A. Cancer statistics, 2019. CA Cancer J Clin. 2019;69(1):7-34.

2. Chen W, Zhou S, Mao L, Zhang H, Sun D, Zhang J, et al. Crosstalk between TGF-beta signaling and miRNAs in breast cancer metastasis. Tumour Biol. 2016;37(8):10011-9.

3. McAllister SD, Murase R, Christian RT, Lau D, Zielinski AJ, Allison J, et al. Pathways mediating the effects of cannabidiol on the reduction of breast cancer cell proliferation, invasion, and metastasis. Breast Cancer Res Treat. 2011;129(1):37-47.

4. Qin S, Ma F, Chen L. Gene regulatory networks by transcription factors and microRNAs in breast cancer. Bioinformatics. 2015;31(1):76-83.

5. Lambert M, Jambon S, Depauw S, David-Cordonnier MH. Targeting transcription factors for cancer treatment. Molecules. 2018;23(6):1479.

6. Xie Y, Li L, Wang X, Qin Y, Qian Q, Yuan X, et al. Overexpression of $\mathrm{Cdx2}$ inhibits progression of gastric cancer in vitro. Int J Oncol. 2010;36(2):509-16.

7. Wang HB, Wei H, Wang JS, Li L, Chen AY, Li ZG. Down-regulated expression of LINC00518 prevents epithelial cell growth and metastasis in breast cancer through the inhibition of CDX2 methylation and the Wnt signaling pathway. Biochim Biophys Acta Mol Basis Dis. 2019;1865(3):708-23.

8. Jiang L, Yu X, Ma X, Liu H, Zhou S, Zhou X, et al. Identification of transcription factor-miRNA-IncRNA feed-forward loops in breast cancer subtypes. Comput Biol Chem. 2019;78:1-7.

9. Hu X, Guo J, Zheng L, Li C, Zheng TM, Tanyi JL, et al. The heterochronic microRNA let-7 inhibits cell motility by regulating the genes in the actin cytoskeleton pathway in breast cancer. Mol Cancer Res. 2013;11(3):240-50.

10. Liu X, Cao M, Palomares M, Wu X, Li A, Yan W, et al. Metastatic breast cancer cells overexpress and secrete miR-218 to regulate type I collagen deposition by osteoblasts. Breast Cancer Res. 2018;20(1):127.

11. Wang Q, She Y, Bi X, Zhao B, Ruan X, Tan Y. Dexmedetomidine protects $P C 12$ cells from lidocaine-Induced cytotoxicity through downregulation of COL3A1 mediated by miR-let-7b. DNA Cell Biol. 2017;36(7):518-28.

12. Halsted KC, Bowen KB, Bond L, Luman SE, Jorcyk CL, Fyffe WE, et al. Collagen alpha1(XI) in normal and malignant breast tissue. Mod Pathol. 2008;21(10):1246-54.

13. Karaglani M, Toumpoulis I, Goutas N, Poumpouridou N, Vlachodimitropoulos D, Vasilaros S, et al. Development of novel real-time PCR methodology for quantification of COL11A1 mRNA variants and evaluation in breast cancer tissue specimens. BMC Cancer. 2015;15:694.

14. Al Kubaisy E, Arafat K, De Wever O, Hassan AH, Attoub S. SMARCAD1 knockdown uncovers its role in breast cancer cell migration, invasion, and metastasis. Expert Opin Ther Targets. 2016;20(9):1035-43.

15. Zhou S, He Y, Yang S, Hu J, Zhang Q, Chen W, et al. The regulatory roles of IncRNAs in the process of breast cancer invasion and metastasis. Biosci Rep. 2018;38(5):BSR20180772.

16. Harris TJ, McCormick F. The molecular pathology of cancer. Nat Rev Clin Oncol. 2010;7(5):251-65.

17. Platet N, Hinkel I, Richert L, Murdamoothoo D, Moufok-Sadoun A, Vanier M, et al. The tumor suppressor CDX2 opposes pro-metastatic 
biomechanical modifications of colon cancer cells through organization of the actin cytoskeleton. Cancer Lett. 2017;386:57-64.

18. Tagawa T, Haraguchi T, Hiramatsu H, Kobayashi K, Sakurai K, Inada K, et al. Multiple microRNAs induced by $\mathrm{Cd} \times 1$ suppress $\mathrm{Cd} \times 2$ in human colorectal tumour cells. Biochem J. 2012:447(3):449-55.

19. Encarnacion J, Ortiz C, Vergne R, Vargas W, Coppola D, Matta JL. High DRC levels are associated with Let-7b overexpression in women with breast cancer. Int J Mol Sci. 2016;17(6):865.

20. Lu PW, Li L, Wang F, GU YT. Effects of long non-coding RNA HOST2 on cell migration and invasion by regulating microRNA let-7b in breast cancer. J Cell Biochem. 2018;119(6):4570-80.

21. Ma L, Li GZ, Wu ZS, Meng G. Prognostic significance of let-7b expression in breast cancer and correlation to its target gene of BSG expression. Med Oncol. 2014;31(1):773.

22. He JH, Han ZP, Zou MX, He ML, Li YG, Zheng L. CDX2/mir-145-5p/ SENP1 pathways affect LNCaP cells invasion and migration. Front Oncol. 2019;9:477.
23. Kim H, Watkinson J, Varadan V, Anastassiou D. Multi-cancer computational analysis reveals invasion-associated variant of desmoplastic reaction involving INHBA, THBS2 and COL11A1. BMC Med Genomics. 2010;3:51.

24. Freire J, Dominguez-Hormaetxe S, Pereda S, De Juan A, Vega A, Simon L, et al. Collagen, type XI, alpha 1: an accurate marker for differential diagnosis of breast carcinoma invasiveness in core needle biopsies. Pathol Res Pract. 2014;210(12):879-84.

\section{Publisher's Note}

Springer Nature remains neutral with regard to jurisdictional claims in published maps and institutional affiliations.
Ready to submit your research? Choose BMC and benefit from:

- fast, convenient online submission

- thorough peer review by experienced researchers in your field

- rapid publication on acceptance

- support for research data, including large and complex data types

- gold Open Access which fosters wider collaboration and increased citations

- maximum visibility for your research: over $100 \mathrm{M}$ website views per year

At BMC, research is always in progress.

Learn more biomedcentral.com/submissions 